\title{
Quantile-based versus Sobol sensitivity analysis in limit state design
}

KALA, Z.

\section{Structures}

2020, vol. 28, December 2020, pp. 2424-2430

ISSN: 2352-0124

DOI: https://doi.org/10.1016/j.istruc.2020.10.037

Accepted manuscript 


\title{
Quantile-based versus Sobol sensitivity analysis in limit state design
}

\author{
Highlights \\ - $R_{d}$ as the design quantile of resistance $(R)$ of a steel beam under lateral-torsional buckling. \\ - Quantile-oriented sensitivity analysis (QSA) subordinated to contrasts between $R$ and $R_{d}$. \\ - Comparison of QSA with classic Sobol sensitivity analysis (SSA). \\ - Identification of similar sensitivity ranking of input random variables by QSA and SSA. \\ - Different interaction effects are obtained from QSA (strong) and SSA (weak).
}

\begin{abstract}
In limit state design, the reliability of building constructions is generally verified using design quantiles. The design resistance of a structure is explicitly expressed as a low quantile of the cumulative distribution function of resistance. The aim of this article is to show the connections and differences between quantile-oriented sensitivity analysis subordinated to a contrast and classic Sobol sensitivity analysis. Changing the fixed input variable causes synchronous change in the quantile and mean value, but how do the results of these two sensitivity analyses differ? The question is whether or not the changes around the design quantile (measured by contrast indices) are similar to the changes around the mean value, which are measured using Sobol's indices. Comparison is performed on a case study, where the resistance of the structure is expressed by a non-linear function, the inputs of which are random material and geometric characteristics of the structure. The nondimensional slenderness is a deterministic parameter, which changes the influence of input variables on the resistance as the model output. It was concluded upon comparing the results of both sensitivity analyses that the rank of the most important variables is the same for both low and high slenderness and is similar for intermediate slenderness. However, the interaction effects are very different. The identification of insignificant variables is the same. Other significant similarities and differences between both types of sensitivity analyses are presented in the article.
\end{abstract}

Keywords: Sensitivity analysis; structural reliability; Eurocodes; design quantiles; stability; buckling; steel

\section{Introduction}

Eurocodes [1] assess the reliability of building structures using design quantiles of load and resistance, which are more practical for structural design than failure probability [2][3]. The basic reliability targets for design values in ultimate limit states recommended in [1] are based on a semi-probabilistic approach [4][5]. Structural design is reliable if the design resistance (lower quantile) is higher than the design load (upper quantile). This concept evaluates reliability using two states, i.e., satisfactory (design resistance $>$ design load) or unsatisfactory (design resistance $<$ design load). From a design point of view, this reliability assessment is sufficient even without the analysis of the failure probability $P_{f}$ or the reliability index $\beta$ [1]. A structure is either designed according to standards and is therefore sufficiently reliable, or such a design must be ensured. Economically and reliably optimal design means that the design resistance is equal to the design load. Higher reliability than is prescribed by standards is possible, however, higher economic costs of construction would be required (material, etc.).

Reviews of sensitivity methods in interdisciplinary contexts are offered in [6], [7], [8] and [9]. In terms of utilization in the construction industry, the methods of sensitivity analysis (SA) can be divided into global sensitivity analysis [10], [11], [12], [13], etc., reliability-oriented sensitivity analysis ROSA [14], [15], [16], [17], etc., and other specific types of SA, see, for e.g., [18], [19], [20] and [21]. The first types of analyses focus on the distribution or certain moments of the output function while ROSA considers $P_{f}, \beta$ or design quantiles as the 
quantity of interest. Comparative case studies [22] have shown that the differences between indices in the first and second group as well as between the groups are large and the information value of ROSA indices is unclear.

In civil engineering, SA methods are often applied in a conventional manner, which takes advantage of the knowledge that the methods of the first group are partially empathic to reliability, see, for e.g., [23] and [24]. It can be argued that the input variable (imperfection) could have an influence on the distribution of the limit state function but not on $P_{f}$ and conversely. However, such cases are not common in engineering practice. The results of reliability analysis tend to be more or less consistent with the results of Sobol sensitivity analysis (SSA), see, for e.g., analyses of the failure mode and load of steel frames [25].

The question is, what is the information value of the indices of the first group if the reliability analysis is the main subject of interest? Intuitively, it can be surmised that when the input variable influences the variance, it can also have some influence on structural reliability. A basic comparison of SA methods in terms of $P_{f}$ has been performed in [22]. In this article, we will focus on the random resistance $R$, for which we will evaluate the SA of the design quantile of the resistance $R_{d}$ together with SSA. The structural resistance $R$ represents the model output, which is generally non-linearly dependent on the inputs. The aim of this article is to show the connections between quantile-oriented sensitivity analysis subordinated to a contrast [14] (referred to as QSA in this article) and classic SSA. The results of QSA are compared with the results of SSA using a simple case study, where the quantity is used in limit state design (LSD).

This article examines whether or not the change in $R$ that occurs around the design quantiles is similar to the change around the mean value, which is measured by Sobol indices. The design resistance is explicitly expressed as the $\alpha^{\text {th }}$ quantity from the cumulative distribution function of $R$. The subject of interest is the evaluation of inputs contributing to the extreme output values. It can be noted that the link between quantile-based SA and Sobol main effect sensitivity indices has already been studied in [26], however, not with the use of GSA [14], which is applied here. The GSA applied here is based on contrasts that measure the average distance between the quantile and the output in comparison to the measures proposed in [26], which use the average distance between the quantities. Additional studies of quantile-oriented SA subordinated to a contrast can be found, for e.g., in [27], [28] and [29].

\section{Sensitivity measures based on quantiles of output}

The model is represented by a mapping $f$ which relates the domain of inputs to the output space:

$$
R=f\left(X_{1}, X_{2}, \ldots, X_{M}\right),
$$

where $R$ is the model output (static resistance) and input variables $\left(X_{1}, X_{2}, \ldots, X_{M}\right)$ are described using probability density functions (pdfs), which reflect the uncertain knowledge of the system under analysis.

The Goal Oriented Sensitivity Analysis is based on contrast functions [14]. The contrast function $\psi$ associated with $\alpha$-quantile can be written using parameter $\theta$ as:

$$
\psi(\theta)=E(\psi(R, \theta))=E\left((R-\theta)\left(\alpha-1_{R<\theta}\right)\right) .
$$

The estimator of $\alpha$-quantile $\theta^{*}$ is given as $\theta^{*}=\operatorname{Argmin} \psi(\theta)$. Based on [14] the first-order quantile contrast index $Q_{i}$ (first-order or main sensitivity index subordinated to the contrast) can be defined as:

$$
Q_{i}=\frac{\min _{\theta} \psi(\theta)-E\left(\min _{\theta} E\left(\psi(R, \theta) \mid X_{i}\right)\right)}{\min _{\theta} \psi(\theta)}
$$

where the numerator is the contrast variation due to $X_{i} . Q_{i}$ is the sensitivity index of the estimator of $\theta^{*}$. The minimum value of contrast $\psi(\theta)$ can be calculated, for e.g., using $N$ runs of the Latin Hypercube Sampling method (LHS) [30] and [31] as:

$$
\min _{\theta} \psi(\theta) \approx \frac{1}{N} \sum_{n=1}^{N}\left(f\left(X_{1}(n), X_{2}(n), \ldots, X_{M}(n)\right)-\theta^{*}\right)\left(\alpha-1_{f\left(X_{1}(n), X_{2}(n), \ldots, X_{M}(n)\right) \leq \theta^{*}}\right)
$$

where $\theta^{*}$ is the estimator of $\alpha$-quantile. The $\alpha$-quantile $\theta^{*}$ can be estimated from LHS runs so that $\alpha \cdot N$ runs of $R$ are smaller than $\theta^{*}$ and $(1-\alpha) \cdot N$ runs of $R$ are greater than $\theta^{*}$, see the example shown in Fig. 1 . For example, the estimate of 0.1-percentile (0.001-quantile) is practically calculated as the $400^{\text {th }}$ smallest value in the set arranged in an ascending order with $N=400000$ runs of LHS, see Fig. 1. 


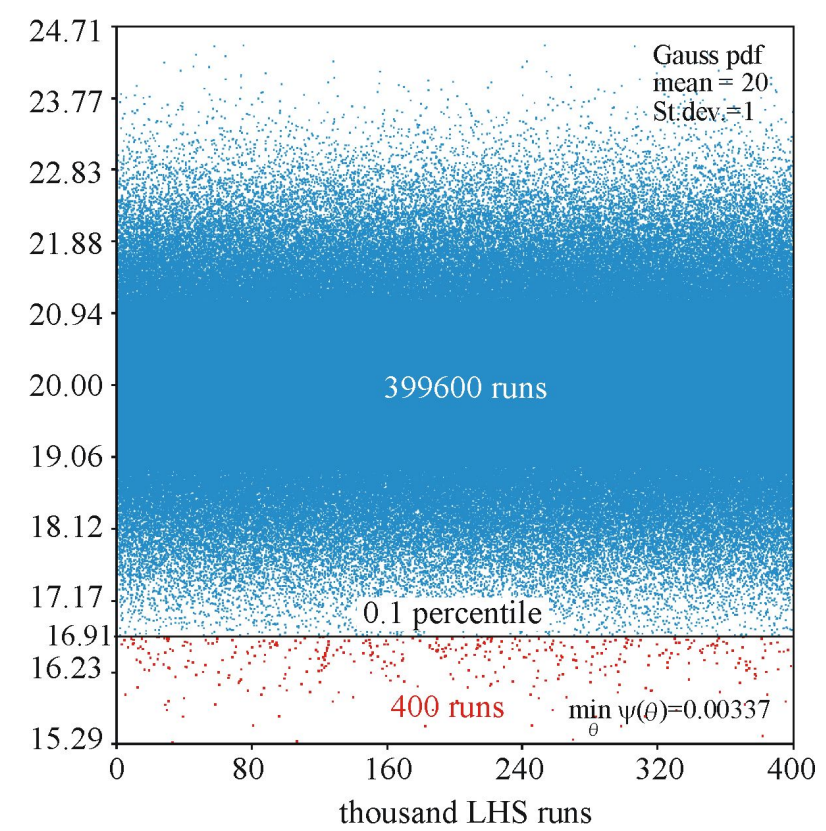

Fig. 1. Example of the estimation of the 0.1-percentile from 400000 runs of LHS.

The second member in the numerator (3) can be determined using two sets of the LHS method. $K$ random realizations of $X_{i}$, i.e., $X_{i}(1), \ldots, X_{i}(k), \ldots, X_{i}(K)$ are generated in the first set. Then $N$ random realizations of the vector $X_{\sim i}$ are generated for each realization $X_{i}(k), k=1, \ldots, K$ (all variables but $X_{i}$ ). For fixed $X_{i}$ we can calculate

$$
\min _{\theta} E\left(\psi(R, \theta) \mid X_{i}\right) \approx m(k)=\frac{1}{N} \sum_{n=1}^{N}\left(f\left(X_{i}(k), X_{\sim i}(k, n)\right)-\theta^{*}(k)\right)\left(\alpha-1_{f\left(X_{i}(k), X_{\sim i}(k, n)\right) \leq \theta^{*}(k)}\right)
$$

where conditional $\alpha$-quantile $\theta^{*}(n)$ is evaluated analogously as in (4) with the difference that the runs of $R$ are obtained for $N$ random realizations of variables $X_{\sim i}$ and fixed (non-random) $X_{i}$. For $K$ runs of $X_{i}$ we then obtain

$$
E\left(\min _{\theta} E\left(\psi(R, \theta) \mid X_{i}\right)\right)=\frac{1}{K} \sum_{k=1}^{K} m(k)
$$

The second-order quantile contrast index $Q_{i j}$ is defined as:

$$
Q_{i j}=\frac{\min _{\theta} \psi(\theta)-E\left(\min _{\theta} E\left(\psi(R, \theta) \mid X_{i}, X_{j}\right)\right)}{\min _{\theta} \psi(\theta)}-Q_{i}-Q_{j}
$$

The higher-order quantile contrast indices can be expressed analogously. The sum of all sensitivity indices must be equal to 1 .

$$
\sum_{i} Q_{i}+\sum_{i} \sum_{j>i} Q_{i j}+\sum_{i} \sum_{j>i} \sum_{k>j} Q_{i j k}+\ldots+Q_{123 \ldots M}=1
$$

The sum of all indices is $2^{M}$-1. If we substitute (2) with the contrast function $\psi(\theta)=E(R-\theta)^{2}$, then the equations presented above lead to the classic Sobol decomposition [10] and [11], where the first-order sensitivity index $S_{i}$ is defined as:

$$
S_{i}=\frac{V(R)-E\left(V\left(R \mid X_{i}\right)\right)}{V(R)}=\frac{V\left(E\left(R \mid X_{i}\right)\right)}{V(R)}=\operatorname{corr}^{2}\left(R, E\left(R \mid X_{i}\right)\right)
$$

where corr is Pearson correlation coefficient. Sobol higher-order sensitivity indices $S_{i j}$, $S_{i j k}$, etc. are determined analogously. It can be noted that (2) is not of quadratic type as the contrast associated with Sobol. 
The substitution of corr with Spearman's rank correlation or Kendall's $\tau$ also leads to the decomposition, where the sum of all indices is 1 . However, the size of indices is generally a little different and their significance may be discussed.

The paper [14] is the first step towards a generalized theory of sensitivity analysis, which views Sobol indices as a special case that is based on quadratic contrast. Analogously as in SSA [32] we can introduce the socalled total index in QSA as:

$$
Q_{T i}=1-\frac{\min _{\theta} \psi(\theta)-E\left(\min _{\theta} E\left(\psi(R, \theta) \mid X_{\sim i}\right)\right)}{\min _{\theta} \psi(\theta)}
$$

The total effect index measures the total contribution to the output contrast due to variable $X_{i}$, i.e., its firstorder effect including all higher-order effects due to interactions. The total effect is given as the sum of all terms in (8) where variable $X_{i}$ is considered. As will be shown below, the introduction of (10) is very useful if the number of input variables is high and the higher-order effects due to interactions are noticeable.

\section{The target reliability and design quantiles}

The aim of this article is QSA of design resistance $R_{d}$, which is calculated as 0.1 percentile of the static resistance $R$. The calculation of 0.1 percentile is based on the semi-probabilistic approach of standard [1]. For a simple case of Gaussian distributed variables of $F$ on the load action side and $R$ on the resistance side [33], the limit state function can be written as:

$$
G=R-F \geq 0
$$

where $F$ and $R$ are statistically independent variables for which we can write:

$$
\mu_{\mathrm{G}}=\mu_{\mathrm{R}}-\mu_{\mathrm{F}}, \sigma_{\mathrm{G}}=\sqrt{\sigma_{\mathrm{R}}^{2}+\sigma_{\mathrm{F}}^{2}}
$$

where $\mu$ is the mean value and $\sigma$ is the standard deviation. The reliability index $\beta$ can be evaluated in relation to the probability of failure $P_{f}$ as:

$$
\beta=\frac{\mu_{\mathrm{G}}}{\sigma_{G}} \text { with } P_{\mathrm{f}}=1-\Phi(\beta)=\Phi(-\beta)
$$

where $\Phi(\bullet)$ is the cumulative normalized Gauss distribution. For the reference period of 50 years, the target reliability recommended in [1] for ultimate limit state is $\beta_{d}=3.8, P_{f}=7.2 \mathrm{E}-5$, see Table C2 in [1] and/or [34]. In order to answer the question of which value on the load action side and which value on the resistance side result in the desired level of reliability, we have to introduce weighting factors $\alpha_{F}$ (for the load action effect) and $\alpha_{R}$ (for the resistance):

$$
\alpha_{F}=\frac{\sigma_{F}}{\sigma_{G}}, \alpha_{R}=\frac{-\sigma_{R}}{\sigma_{G}}
$$

The so-called design values $F_{d}$ and $R_{d}$ can then be calculated as:

$$
\begin{aligned}
& F_{d}=\mu_{\mathrm{F}}+\alpha_{\mathrm{F}} \beta_{\mathrm{d}} \sigma_{\mathrm{F}} \\
& R_{d}=\mu_{\mathrm{R}}+\alpha_{\mathrm{R}} \beta_{\mathrm{d}} \sigma_{\mathrm{R}}
\end{aligned}
$$

If the design is reliable, it must hold that $F_{d} \leq R_{d}$. The probability that resistance $R \leq R_{d}$ can be written as:

$$
P\left(R \leq R_{d}\right)=\Phi\left(\frac{\mu_{\mathrm{R}}-\alpha_{\mathrm{R}} \beta_{\mathrm{d}} \sigma_{\mathrm{R}}-\mu_{\mathrm{R}}}{\sigma_{\mathrm{R}}}\right)=\Phi\left(-\alpha_{\mathrm{R}} \beta_{\mathrm{d}}\right)
$$

Upon substitution of $\beta_{d}=3.8$ into (16) we obtain a probability of 0.001183 , which is approximately 0.1 percentile of $R$, see Fig. 2. An alternative equation to (15) is listed in [1] for variables, which do not have a 
Gaussian distribution, but have a different statistical distribution, such as Gumbel or Log-Normal pdfs. In this article $R_{d}$ is calculated in all cases as 0.1 percentile non-parametrically based on LHS simulations, see Fig. 1.

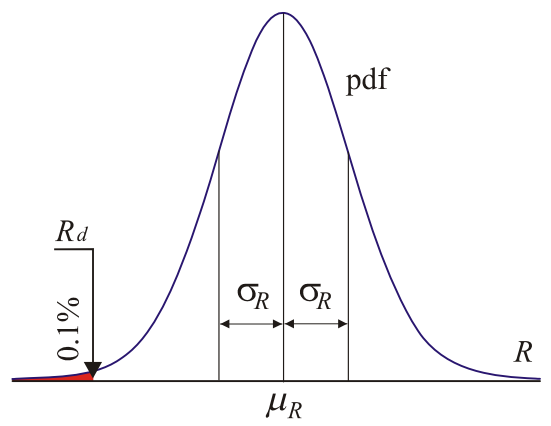

Fig. 2. The random and design resistance.

\section{The computation of static resistance}

The elastic load carrying capacity $M_{R}$ was derived in [35], see also [36]. In this article the computational model of elastic load carrying capacity is taken from [35] with the difference that instead of $M_{R}$ we introduce the symbol $R$, so that we are consistent with the model output (resistance $R$ ) in the preceding paragraphs. The relatively fast response of the non-linear function (17) in the closed-form permits the use of high numbers of runs of the LHS method and gives accurate and highly transparent results even without using metamodels and supercomputers.

The resistance $R$ (17) of an I-beam decreases with its increasing length $L$ due to the LTB phenomena [35]. The initial axial deformation increases when the loading bending moment $M$ increases, see Fig. 3. Failure occurs when the longitudinal stress reaches the yield strength $f_{y}$, which occurs when $M$ approaches $R$ at $x=L / 2$.

$$
\begin{aligned}
& R=-\frac{\sqrt{\left(4 Q_{1}^{2}+4 Q_{1}\left(Q_{4}-2 M_{c r} Q_{3}\right)+Q_{4}^{2}+4 M_{c r} Q_{4} Q_{2}+4 M_{c r}^{2} Q_{2}^{2}\right)}}{4 M_{c r} W_{z}} \\
& +\frac{2 Q_{1}+Q_{4}+2 M_{c r} Q_{2}}{4 M_{c r} W_{z}}
\end{aligned}
$$

where

$$
\begin{aligned}
& Q_{1}=f_{y} M_{c r} W_{y} W_{z} \\
& Q_{2}=M_{c r} W_{z}+P_{z}\left|a_{v 0}\right| W_{y} \\
& Q_{3}=M_{c r} W_{z}-P_{z}\left|a_{v 0}\right| W_{y} \\
& Q_{4}=h P_{z}^{2}\left|a_{v 0}\right| W_{y} \\
& a_{v 0}=e_{0} 2 M_{c r} /\left(2 M_{c r}+h P_{z}\right) \\
& P_{z}=\pi^{2} E I_{z} / L^{2} \\
& W_{y}=2 I_{y} / h \\
& W_{z}=2 I_{z} / b
\end{aligned}
$$

The significance of all variables in equations (17) and (18) are explained in detail in [35]. The input variables are referred to as imperfections in engineering terminology [37]. Generally, these deficiencies arise during the production of the structure, its assembly and subsequent use and have an influence on the resistance, especially in cases where the structure is exposed to loss of stability, such as flexural or lateral-torsional buckling [38]. 
The resistance $R(17)$ is in complete agreement with the elastic load carrying capacity obtained from the computational model created using the software Ansys, which was verified for $0 \leq \bar{\lambda}_{L T} \leq 2.1$ [39] and [40], where $\bar{\lambda}_{L T}$ is the non-dimensional slenderness [41].

The calculation of the second moment of area is based on an idealized geometry of a doubly-symmetric IPN200 section, see Fig. 3. The random variabilities of geometric characteristics $h, b, t_{l}$ and $t_{2}$ (geometric imperfections) of the IPN200 section are introduced according to experimental researches [42] and [43]. The equation for the elastic critical moment $M_{c r}$ is introduced according to [35] and [34], where shear modulus is $G=0.5 \cdot \mathrm{E} /(1+v)$.

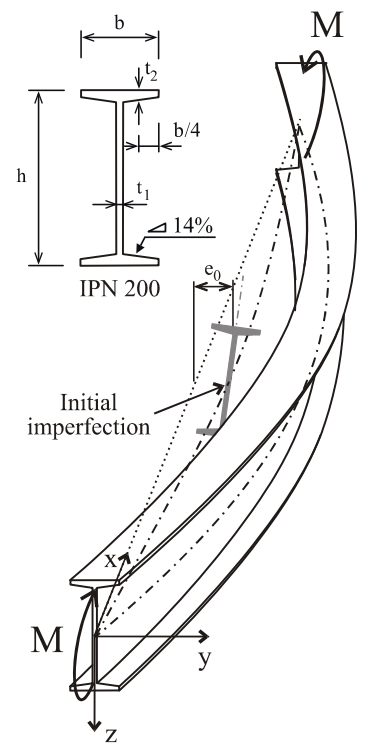

Fig. 3. Lateral-torsional buckling and geometric imperfections.

The shape of initial geometric imperfections is introduced according to the first eigenmode (bow imperfection) of LTB, see Fig. 3. The random variability of amplitude $e_{0}$ is based on the assumption that 5\% of random samples of $e_{0}$ lie outside the tolerance limits $\pm L / 1000$, see, for e.g., [35] and [34]. Statistical characteristics of yield strength are considered according to [42] for steel S235 and [44] for steel S355, see also the discussion in [45]. The statistical characteristics of the other random imperfections in Table 1 are justified in [46]. The influence of residual stress was neglected. All random variables listed in Table 1 are statistically independent.

Table 1. Statistical characteristics of input imperfections [42] [44]

\begin{tabular}{cclccc}
\hline No. & Symbol & Characteristic & Density & Mean $\mu$ & Standard deviation $\sigma$ \\
\hline 1. & $t_{2}$ & Flange thickness & Gauss & $11.3 \mathrm{~mm}$ & $0.518 \mathrm{~mm}$ \\
2. & $f_{y}$ & Yield strength, S235 & Gauss & $297.3 \mathrm{MPa}$ & $16.8 \mathrm{MPa}$ \\
& & Yield strength, S355 & Gauss & $393.8 \mathrm{MPa}$ & $22 \mathrm{MPa}$ \\
3. & $E$ & Modulus of elasticity & Gauss & $210 \mathrm{GPa}$ & $10 \mathrm{GPa}$ \\
4. & $e_{0}$ & Initial curvature, S235 & Gauss & 0 & $L_{1} / 1960 *$ \\
& & Initial curvature, S355 & Gauss & 0 & $L_{2} / 1960 *$ \\
5. & $h$ & Cross-section height & Gauss & $200 \mathrm{~mm}$ & $0.885 \mathrm{~mm}$ \\
6. & $b$ & Flange width & Gauss & $90 \mathrm{~mm}$ & $0.888 \mathrm{~mm}$ \\
7. & $t_{l}$ & Web thickness & Gauss & $7.5 \mathrm{~mm}$ & $0.293 \mathrm{~mm}$ \\
8. & $v$ & Poisson's ratio & Gauss & 0.3 & 0.009 \\
& & & & &
\end{tabular}

where $L_{1} \approx 2.15 \bar{\lambda}_{L T}-0.75 \bar{\lambda}_{L T}^{2}+1.95 \bar{\lambda}_{L T}^{3}-0.39 \bar{\lambda}_{L T}^{4}$ for S235 [46],

$$
L_{2} \approx 1.7 \bar{\lambda}_{L T}-0.36 \bar{\lambda}_{L T}^{2}+0.95 \bar{\lambda}_{L T}^{3}-0.15 \bar{\lambda}_{L T}^{4} \text { for S355. }
$$

Dependencies between $L_{1}$ and $\bar{\lambda}_{L T}, L_{2}$ and $\bar{\lambda}_{L T}$ listed in Table 1 approximate the procedure in [41]. The analytical function between $L$ and $\bar{\lambda}_{L T}$ is derived in [47]. 
Slenderness is one of the basic parameters in the design of steel structures. The parametric study uses $\bar{\lambda}_{L T}$ to evaluate the effect of parametric change of $\bar{\lambda}_{L T}$ on sensitivity indices, where $\bar{\lambda}_{L T}$ changes with the step of 0.01 . The length of the member $L$ is assigned according to steel grade as $L_{1}$ or $L_{2}$.

\section{Sensitivity analysis results}

The LHS method is used to evaluate all sensitivity indices. Each index $Q_{i}(3)$ is evaluated using $K=4000$ runs of $m(j)$ (5). Each realization of $m(j)$ is evaluated using a set with $N=400000$ runs of $R$, in which $\theta^{*}(j)$ represents the conditional 0.1 percentile, which is the $400^{\text {th }}$ smallest value in the set arranged in an ascending order, see, for e.g., Fig. 1. The denominator in (3) is calculated according to (4) for $N=400000$ runs. Indices $S_{i}, Q_{i j}, Q_{i j k}, Q_{i j k l}$ and $Q_{i T}$ are calculated analogously using the same values $K$ and $N$.

\subsection{QSA for 8 input imperfections from Table 1}

QSA with $M=8$ imperfections (Table 1) leads to $2^{8}-1=255$ quantile contrast indices, which is too much for practical calculation. Therefore, all global effects are described using indices $Q_{i}$ and $Q_{T}$, see Fig. 4, Fig. 5 and Fig. 6. The total effect indices $Q_{T i}$ provide a cumulative measure of sensitivity inclusive of interaction effects of any order.

The difference $Q_{T i}-Q_{i}$ is a measure of the extent to which the $i^{\text {th }}$ imperfection is involved in interactions with any other input imperfection. The plots of $Q_{T i}$ and $Q_{i}$ are similar in shape, but sizes and mutual proportions are different, see Fig. 4 and Fig. 5. In contrast to $Q_{i}$, the total effects $Q_{T i}$ show greater involvement of $t_{2}$ for low slenderness and $E$ for high slenderness. The modulus of elasticity $E$ is a completely non-influential imperfection for $\bar{\lambda}_{L T} \leq 0.4$ (see Fig. 5) and $f_{y}$ is a completely non-influential imperfection for approximately $\bar{\lambda}_{L T} \geq 5$ (see Fig. $6)$.

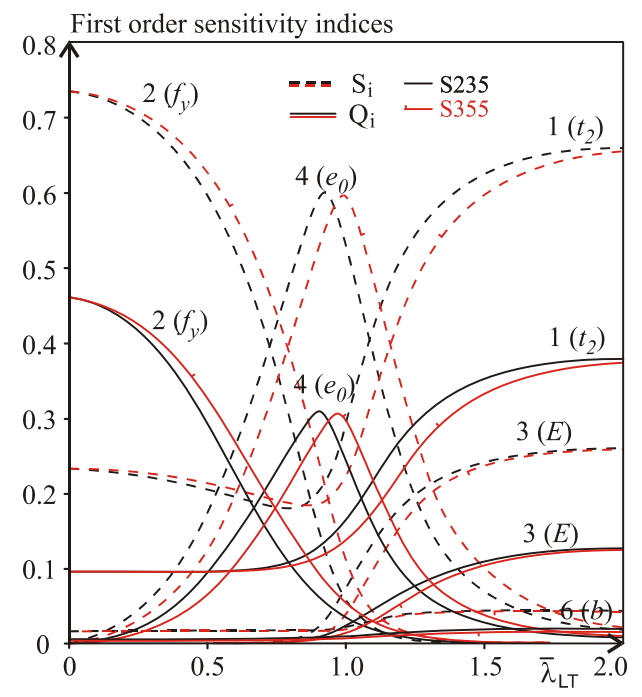

Fig. 4. First-order sensitivity indices $S_{i}, Q_{i}$ for $M=8$.

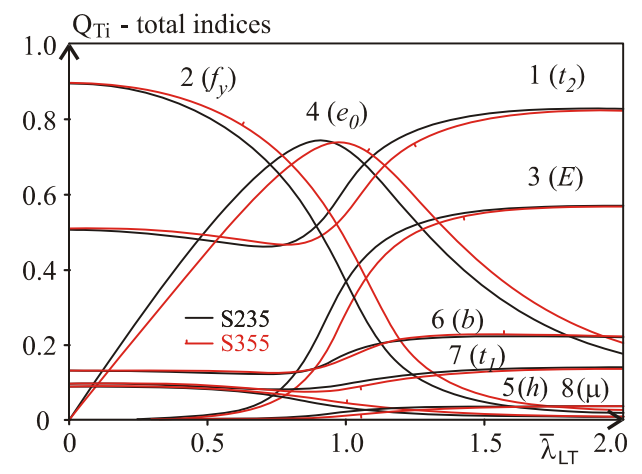

Fig. 5. Total quantile contrast indices for $M=8$. 
The plots of $Q_{i}$ and $S_{i}$ are similar in shape. However, Sobol indices $S_{i}$ are significantly higher than $Q_{i}$, see Fig. 4. This means that QSA identifies more significant interaction effects than SSA. SSA confirms approximately zero higher-order interactions $1-\sum_{\mathrm{i}} S_{i} \approx 0$ [35], which is in sharp contrast to the results of QSA, where the difference $Q_{T i}-Q_{i}$ identifies strong interaction effects. QSA confirms the results of SSA [35] in the identification of marginal main effects of imperfections $h, b, t_{l}$ and $v$.

The plots of $S_{i}$ in Fig. 4 (IPN 200) are almost identical to previous results of SSA of the resistance of beam IPE 220 [35]. It can be noted that beams IPN 200 and IPE 220 [35] have the same variation coefficients of imperfections $h, b, t_{1}, t_{2}$ and probability models of all other imperfections are the same in the case of steel S235 [45]. Sobol sensitivity indices $S_{i}$ are the same for both sections under these conditions.

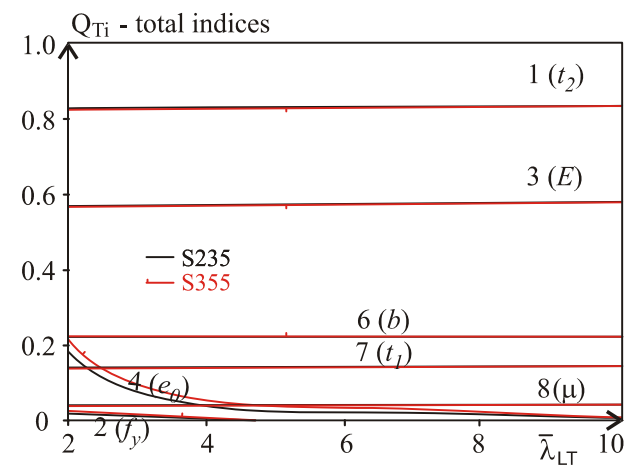

Fig. 6. Total quantile contrast indices for $M=8$.

It is apparent from Fig. 4 and Fig. 5 that the plots of sensitivity indices of steel S355 (red curves) approximately follow the plots of sensitivity indices of steel S235 (black curves), however, with the difference that the red curves are slightly shifted to the right, which is particularly noticeable in beams with intermediate slenderness. The values of indices $Q_{5}, Q_{7}$ and $Q_{8}$, which are lower than 0.03 and are not shown in Fig. 4. The shift to the right is due to the fact that losses (contrasts) between $R$ and $R_{d}$ of members of steel S355 are more influenced by the variability of $f_{y}$ and less influenced by $t_{2}$ and $E$. Indices $Q_{T i}$ of imperfections $h$ and $v$ are very small and these variables can be regarded as non-influential, see Fig. 5 and Fig. 6.

The values of $Q_{T i}$ remain practically unchanged for very high slenderness $\bar{\lambda}_{L T} \geq 2$, with the exception of $Q_{T 2}$ and $Q_{T 4}$, which decrease to zero, see Fig. 6. The values of $Q_{T i}$ and $Q_{i}$ are shown in Fig. 7 for the limit case of $\bar{\lambda}_{L T} \rightarrow \infty$. Practically, $\bar{\lambda}_{L T}=100$ was considered in the calculation. However, sensitivity indices remain constant for $\bar{\lambda}_{L T}>10$. The results in Fig. 7 apply to steel of both steel grades S235 and S355.

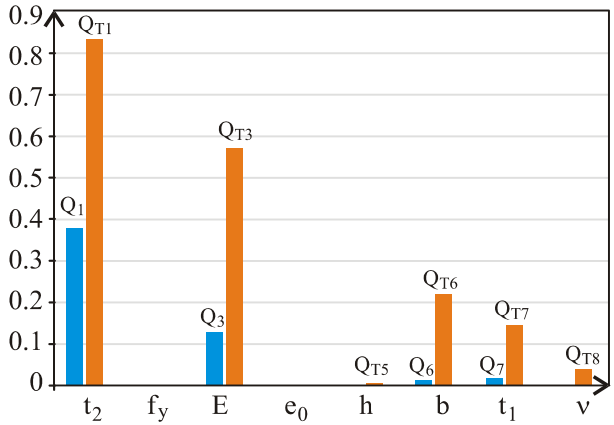

Fig. 7. QSA indices, $\bar{\lambda}_{L T}=100, \mathrm{~S} 235$ and S355.

\subsection{QSA for four input imperfections}

The first four imperfections $t_{2}, f_{y}, E$ and $e_{0}$ listed in Table 1 were identified in the previous chapter as crucial. The comparison of QSA and SSA shows an agreement in the identification of non-influential imperfections $h, b, t_{l}$ and $v$, whose variability can be completely neglected. Such imperfections can be fixed at any value of their domain without influencing the average absolute loss (contrast) between $R$ and $R_{d}$ or $R$ and $\mu_{R}$.

Let us consider the random variability of the first four imperfections $t_{2}, f_{y}, E$ and $e_{0}(M=4)$ and introduce the other four $h, b, t_{1}$ and $v$ as non-random by their mean value. This makes it possible to evaluate all $2^{4}-1=15$ quantile indices in decomposition (8), which can be clearly illustrated; see Fig. 8 and Fig. 9. 


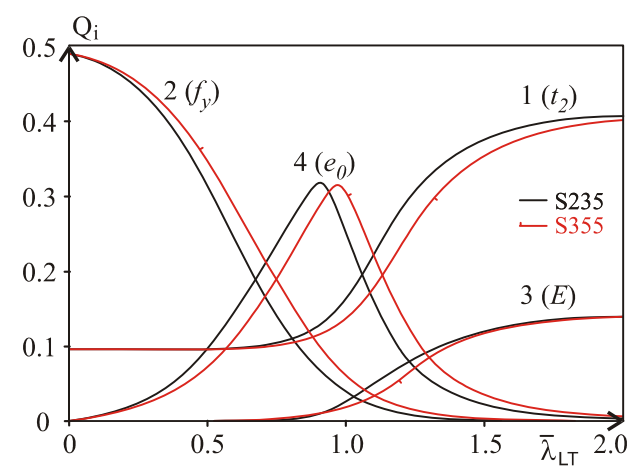

Fig. 8. First-order quantile contrast indices for $M=4$.

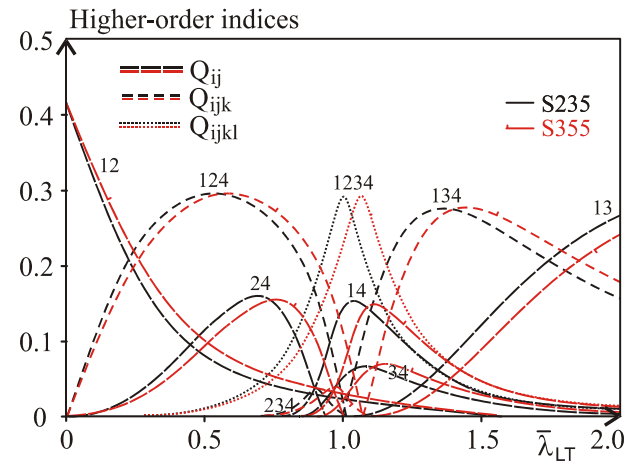

Fig. 9. Higher-order quantile contrast indices for $M=4$.

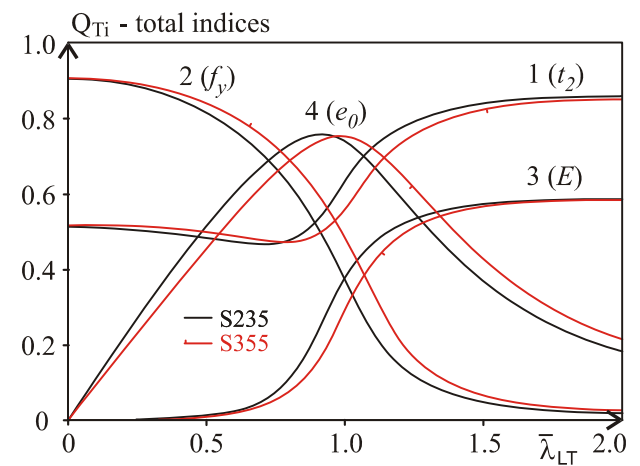

Fig. 10. Total quantile contrast indices for $M=4$.

The total indices $Q_{T i}$ of imperfections $t_{2}, f_{y}, E$ and $e_{0}$ shown in Fig. $10(M=4)$ are approximately the same as the indices in Fig. $5(M=8)$ with the exception of $\bar{\lambda}_{L T}=2$, where $Q_{T i}$ in Fig. 5 is slightly lower than $Q_{T i}$ in Fig. 10. The approximate similarity of indices $Q_{i}$ in Fig. 4 and Fig. 8 also applies. Fixing the values of imperfections $h, b, t_{l}$ and $v$ (setting their mean value), therefore, does not have a significant effect on the main and total effects of imperfections $t_{2}, f_{y}, E$ and $e_{0}$.

The non-zero total effects of imperfections $f_{y}$ and $e_{0}$ are surprisingly also found for high slenderness $\bar{\lambda}_{L T} \geq 2$, see Fig. 6. Decomposition (8) performed for $M=4$ shows that imperfections $f_{y}$ and $e_{0}$ are involved in interactions with imperfections $t_{2}$ and $E$ for $\bar{\lambda}_{L T}=2$ (see Fig. 9), then their influence decreases. Decomposition (8) for limit case $\bar{\lambda}_{L T} \rightarrow \infty$ leads to only three non-zero sensitivity indices, see Fig. 11. 


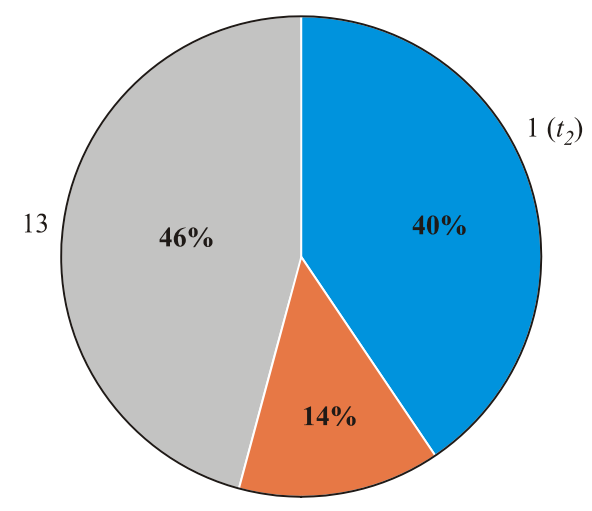

$3(E)$

Fig. 11. QSA indices (8) for $M=4, \bar{\lambda}_{L T}=100, \mathrm{~S} 235$ and S355.

\section{Discussion of the results}

In all cases, it was found that $\operatorname{corr}\left(Q\left(R \mid X_{i}\right), E\left(R \mid X_{i}\right)\right) \approx 1$, where $Q\left(R \mid X_{i}\right)$ is the conditional 0.1 percentile and $E\left(R \mid X_{i}\right)$ is the conditional mean value. Changing the fixed input variable $X_{i}$ causes synchronous change in the quantile and mean value calculated from $X_{\sim i}$. This was also observed when fixing pairs, triples, etc. The differences between indices $Q_{i}$ and $S_{i}$ are mainly due to the different nature of the contrast functions. The contrast function (2) associated with the quantile is based on the average normalized absolute distance between the quantile and $R$, while SSA is based on the average quadratic distance between the mean value and $R$ (variance). Although QSA and SSA lead to the same or similar conclusions, the interaction effects identified by QSA are much less comprehensible.

Although equation (17) represents a typical non-linear function of static resistance $R$ of a steel structural element, the sensitivity analysis results cannot be generalized for all stochastic computational models. The question remains, in which cases could QSA lead to significantly different conclusions than SSA in terms of identifying the values of the input variables?

In the case study presented here, SSA showed almost zero values of higher-order sensitivity indices. This is common when studying the resistance of structural elements [45] and [49], but not the resistance of steel frames (systems), where SSA identified significant contributions of interaction effects between system elements [23] and [50]. Although there is some similarity between the QSA and SSA results presented here, these conclusions cannot be generalized for sensitivity analysis of the resistance of steel frames with slender columns, where loss of stability is a significant phenomenon.

It can be noted that the applied analytical solution makes it possible to study the influence of imperfections even in very slender (long) members. However, verification of the obtained results using FE models of very slender members would be desirable. Unfortunately, the number of finite elements and the computational demand of FE models of long members in simulations is very high and FE models with higher number of input variables exhibit certain numerical inaccuracies [46]. QSA based on FE models can be considered in the future in connection with supercomputers (FE models) and polynomial chaos expansions [48] (metamodels).

Estimation of sensitivity indices using the LHS method can be performed using high performance computing. The second member in the numerator in equation (3) is computed using double-nested-loop simulation of the LHS method, which is highly demanding on CPU time, therefore, it is effective to let the inner loops run as a parallel process. Further optimization may involve the inner loop, where each LHS sample can run as a parallel process as well. The same parallelization of processes can be introduced when estimating other sensitivity indices.

\section{Conclusion}

This article presents the comparison of the results of quantile-oriented sensitivity analysis (QSA) with the results of classic Sobol sensitivity analysis (SSA) using a simple case study. The subject of interest in SSA is the resistance (and its changes around the mean value) while the subject of interest in QSA is the lower quantile of resistance (and changes in resistance around this quantile). The non-dimensional slenderness is a deterministic parameter, which changes the influence of input variables on the resistance as the model output. Increasing the slenderness shifts our attention from material imperfections to geometric imperfections. 
The results of the case study showed that the rank of the most important variables is the same for both low $\left(\bar{\lambda}_{L T}<0.45\right)$ and high $\left(\bar{\lambda}_{L T}>1.35\right)$ slenderness. For low slenderness, the first and second dominant variables are yield strength $f_{y}$ and flange thickness $t_{2}$. For high slenderness, they are flange thickness $t_{2}$ and modulus of elasticity $E$. For intermediate slenderness $\left(0.45 \leq \bar{\lambda}_{L T} \leq 1.35\right)$, the initial curvature $e_{0}$ is the first or second dominant variable alternating the first position with $t_{2}$ or $f_{y}$, where $f_{y}$ decreases its sensitivity rank rapidly with increasing slenderness. These findings were made using both QSA and SSA.

Although the main conclusions of QSA and SSA are approximately the same, each SA reaches its conclusion in a different manner using indices of different types. The conclusions from SSA are made on the basis of first-order indices, while higher-order indices are practically zero. On the contrary, QSA has the sum of first-order indices approximately the same as the sum of higher-order indices and thus the conclusions of QSA are made on the basis of first-order indices and total indices.

In the presented study, SSA leads to similar conclusions as QSA in terms of identifying the most important input variables. The comparison of QSA and SSA also shows an agreement in identifying four totally non-influential imperfections. However, in terms of less or moderately important variables, the conclusions of QSA and SSA may differ. In cases where SSA shows almost zero influence, QSA may show certain small influence. An example is the initial curvature $e_{0}$ for non-dimensional slenderness equal to two. Both SA are in agreement in almost zero first-order indices, but QSA also identifies higher-order indices. It can be noted that imperfection $e_{0}$ is a typical input variable with strong non-linear influence on the static resistance.

It is generally known that the variance is not sufficient for the sensitivity analysis of reliability, but are the results of SSA of such little practical use for the analysis of reliability? The case study presented here showed that the results of SSA are partially empathic to the results of QSA, where the lower quantile is the subject of interest. However, they are not the same.

Although the presented conclusions cannot be generalized for all stochastic computational models, many stochastic computational models of resistance of steel elements may have very similar dependencies between inputs and outputs, including the nature of input random variables. As was shown in the article, change in steel grade has little influence on the results of SA. It is unclear whether any agreement between SSA and QSA can also be obtained in steel frames, where SSA has shown significant contributions of interaction effects between system elements [23].

QSA is proving to be a possible tool for identifying the effects of input imperfections on the contrasts between model outputs and design quantiles. This domain deserves much more work in order to make QSA a useful and practical tool. Future work will be focused on other types of sensitivity indices subordinated to contrasts, which are suitable for further reliability analysis of structures associated with probability distributions.

\section{Acknowledgements}

The work has been supported and prepared within the project namely "Probability oriented global sensitivity measures of structural reliability" of The Czech Science Foundation (GACR, https://gacr.cz/) no.20-01734S, Czechia.

\section{References}

[1] EN 1990, Eurocode-Basic of Structural Design, CEN, Brussels, 2002.

[2] G. Sedlacek, H. Stangenberg, Design philosophy of Eurocodes - background information, Journal of Constructional Steel Research 54 (1) (2000) 173-190.

[3] G. Sedlacek, Ch. Müller, The European standard family and its basis, Journal of Constructional Steel Research 62 (11) (2006) 1047-1059.

[4] G. Sedlacek, O. Kraus, Use of safety factors for the design of steel structures according to the Eurocodes, Engineering Failure Analysis 14 (2007) 434-441

[5] L. Simões da Silva, T. Tankova, L. Marques, On the safety of the European stability design rules for steel members, Structures 8 (2016) 157-169

[6] P. Wei, Z. Lu, J. Song, Variable importance analysis: A comprehensive review, Reliability Engineering and System Safety 142 (2015) 399-432.

[7] E. Borgonovo, E. Plischke, Sensitivity analysis: A review of recent advances, European Journal of Operational Research, 248 (3) (2016) 869-887.

[8] A. Saltelli, K. Aleksankina, W. Becker, P. Fennell, F. Ferretti, N. Holst, S. Li, Q. Wu, Why so many published sensitivity analyses are false: A systematic review of sensitivity analysis practices, Environmental Modelling \& Software 114 (2019) 29-39. 
[9] J. Antucheviciene, Z. Kala, M. Marzouk, E.R. Vaidogas, Solving civil engineering problems by means of fuzzy and stochastic MCDM methods: Current state and future research, Mathematical Problems in Engineering 2015 (2015) 1-16. Article ID 362579

[10] I.M. Sobol', Sensitivity estimates for nonlinear mathematical models, Mathematical Modelling and Computational Experiment 1(4) (1993) 407-414 [Translated from Russian. Sobol', IM. Sensitivity estimates for nonlinear mathematical models. Matematicheskoe Modelirovanie 2 (1) (1990) 112-118.]

[11] I.M. Sobol', Global sensitivity indices for nonlinear mathematical models and their Monte Carlo estimates, Mathematics and Computers in Simulation 55 (1-3) (2001) 271-280.

[12] E. Borgonovo, A new uncertainty importance measure, Reliability Engineering and System Safety 92 (2007) 771-784.

[13] F. Gamboa, T. Klein, A. Lagnoux, Sensitivity analysis based on Cramér-von Mises distance, SIAM/ASA Journal on Uncertainty Quantification 6 (2018) 522-548.

[14] J.-C. Fort, T. Klein, N. Rachdi, New sensitivity analysis subordinated to a contrast, Communications in Statistics - Theory and Methods 45(15) (2016) 4349-4364.

[15] S. Xiao, Z. Lu, Structural reliability sensitivity analysis based on classification of model output, Aerospace Science and Technology 71 (2017) 52-61.

[16] C. Ling, Z. Lu, K. Cheng, B. Sun, An effcient method for estimating global reliability sensitivity indices, Probabilistic Engineering Mechanics 56 (2019) 35-49.

[17] H.O. Madsen, Omission sensitivity factor, Structural Safety 5 (1988) 35-45.

[18] D. Lehký, L. Pan, D. Novák, M. Cao, M. Šomodíková, O. Slowik, A comparison of sensitivity analyses for selected prestressed concrete structures. Structural Concrete 20 (2019) 38-51.

[19] L. Su, T. Wang, H. Li, Y. Cao, L. Wang, Multi-criteria decision making for identification of unbalanced bidding. Journal of Civil Engineering and Management 26(1) (2020) 43-52.

[20] P. Wang, Z. Lu, Z. Tang, A derivative based sensitivity measure of failure probability in the presence of epistemic and aleatory uncertainties. Computers \& Mathematics with Applications 65 (2013) 89-101.

[21] H. Pan, L. Tian, X. Fu, H. Li, Sensitivities of the seismic response and fragility estimate of a transmission tower to structural and ground motion uncertainties. Journal of Constructional Steel Research 167 (2020) Article 105941.

[22] Z. Kala, Sensitivity analysis in probabilistic structural design: A comparison of selected techniques, Sustainability 12 (2020), 4788.

[23] Z. Kala, Geometrically non-linear finite element reliability analysis of steel plane frames with initial imperfections, Journal of Civil Engineering and Management 18(1) (2012) 81-90.

[24] J. Štefaňák, Z. Kala, L. Miča, A. Norkus, Global sensitivity analysis for transformation of Hoek-Brown failure criterion for rock mass, Journal of Civil Engineering and Management 24(5) (2018) 390-398.

[25] S.R. Arwade, M. Moradi, A. Louhghalam, Variance decomposition and global sensitivity for structural systems, Engineering Structures 32 (2010) 1-10.

[26] S. Kucherenko, S. Song, L. Wang. Quantile based global sensitivity measures, Reliability Engineering and System Safety 185 (2019) 35-48.

[27] T. Browne, J.-C. Fort, B. Iooss, L.G. Loïc, Estimation of quantile-oriented sensitivity indices, Hal.archives-ouvertes 2017 Preprint. https://hal.archives-ouvertes.fr/hal-01450891

[28] V. Maume-Deschamps, I. Niang, Estimation of quantile oriented sensitivity indices, Statistics and Probability Letters 134 (2018) 122-127. https://doi.org/10.1016/j.spl.2017.10.019

[29] Z. Kala, Quantile-oriented global sensitivity analysis of design resistance, Journal of Civil Engineering and Management 25(4) (2019) 297-305.

[30] M.D. McKey, W.J. Conover, R.J. Beckman, A comparison of the three methods of selecting values of input variables in the analysis of output from a computer code, Technometrics 21 (2) (1979) 239-245.

[31] R.C. Iman, W.J. Conover, Small sample sensitivity analysis techniques for computer models with an application to risk assessment, Communications in Statistics - Theory and Methods 9 (17) (1980) 17491842.

[32] A. Saltelli, K. Chan, E.M. Scott, Sensitivity Analysis, Wiley Series in Probability and Statistics, John Wiley \& Sons, New York, 2004.

[33] A.M. Freudenthal, Safety and the probability of structural failure. Trans. ASCE 121 (1956) 1337-1397. http://refhub.elsevier.com/S0029-8018(17)30486-9/sref3

[34] Z. Kala, Reliability analysis of the lateral torsional buckling resistance and the ultimate limit state of steel beams with random imperfections, Journal of Civil Engineering and Management 21 (7) (2015) 902-911.

[35] Z. Kala, Sensitivity and reliability analyses of lateral-torsional buckling resistance of steel beams, Archives of Civil and Mechanical Engineering 15 (2015) 1098-1107.

[36] Z. Kala, Elastic lateral-torsional buckling of simply supported hot-rolled steel I-beams with random imperfections, Procedia Engineering 57 (2013) 504-514. 
[37] C. Mercier, A. Khelil, A. Khamisi, F. Al Mahmoud, R. Boissiere, A. Pamies, Analysis of the global and local imperfection of structural members and frames, Journal of Civil Engineering and Management 25(8) (2019) 805-818.

[38] J. Jönsson, M.S. Müller, C. Gamst, J. Valeš, Z. Kala, Investigation of European flexural and lateral torsional buckling interaction, Journal of Constructional Steel Research, 156 (2019) 105-121.

[39] J. Valeš, Z. Kala, J. Martinásek, Solving lateral beam buckling problems by means of solid finite elements and nonlinear computational methods, International Journal of Mathematical and Computational Methods (2016) 103-108.

[40] Z. Kala, J. Valeš, J. Martinásek, Inelastic finite element analysis of lateral buckling for beam structures, Procedia Engineering 172 (2017) 481-488.

[41] ENV 1993-1-1:1992, Eurocode 3: Design of Steel Structures - Part 1.1: General Rules and Rules for Buildings, CEN - European committee for Standardization, Brussels (Belgium), 1992.

[42] J. Melcher, Z. Kala, M. Holický, M. Fajkus, L. Rozlívka, Design characteristics of structural steels based on statistical analysis of metallurgical products, Journal of Constructional Steel Research, 60 (3-5) (2004) 795-808.

[43] Z. Kala, J. Melcher, L. Puklický, Material and geometrical characteristics of structural steels based on statistical analysis of metallurgical products, Journal of Civil Engineering and Management 15 (3) (2009) 299-307.

[44] A. Sadowski, J.M. Rotter, T. Reinke, T. Ummenhofer, Statistical analysis of the material properties of selected structural carbon steels, Structural Safety 53 (2015) 26-35.

[45] Z. Kala, J. Valeš, Imperfection sensitivity analysis of steel columns at ultimate limit state, Archives of Civil and Mechanical Engineering 18(4) (2018) 1207-1218.

[46] Z. Kala, J. Valeš, Stochastic assessment and lateral-torsional buckling design of I-beams, Journal of Constructional Steel Research 139 (2017) 110-122.

[47] J. Valeš, Z. Kala, J. Martinásek, A. Omishore, FE nonlinear analysis of lateral-torsional buckling resistance, International Journal of Mechanics 10 (2016) 235-241.

[48] B. Sudret, Global sensitivity analysis using polynomial chaos expansions. Reliability Engineering and System Safety 93 (2008) 964-979.

[49] Z. Kala, J. Valeš, Global sensitivity analysis of lateral-torsional buckling resistance based on finite element simulations, Engineering Structures 134 (2017) 37-47.

[50] Z. Kala, Global sensitivity analysis in stability problems of steel frame structures, Journal of Civil Engineering and Management 22(3) (2016) 417-424.

\section{List of Figure Captions}

Fig. 1. Example of the estimation of the 0.1-percentile from 400000 runs of LHS

Fig. 2. The random and design resistance.

Fig. 3. Lateral-torsional buckling and geometric imperfections.

Fig. 4. First order sensitivity indices $S_{i}, Q_{i}$ for $M=8$.

Fig. 5. Total quantile contrast indices for $M=8$.

Fig. 6. Total quantile contrast indices for $M=8$.

Fig. 7. QSA indices, $\bar{\lambda}_{L T}=100, \mathrm{~S} 235$ and S355.

Fig. 8. First order quantile contrast indices for $M=4$.

Fig. 9. Higher-order quantile contrast indices for $M=4$.

Fig. 10. Total quantile contrast indices for $M=4$.

Fig. 11. QSA indices (8) for $M=4, \bar{\lambda}_{L T}=100, \mathrm{~S} 235$ and S355. 